\title{
A Comparative Studies of the Activities of Phenoloxidase, Carboxylesterase and Alkaline Phosphatase from Different Geographic Strains of Flacherie-Infected
}

\section{Bombyx mori}

\author{
Wenchu Li, Jingyu Zhang, Shizhang Feng \\ Department of Sericultural Science, College of Animal Science, South China Agricultural \\ University, Guangzhou, China.
}

\begin{abstract}
:
Flacherie diseases of silkworm caused by complex pathogens but mainly Bacillus spp. in springtime. The paper aims to explore interaction between different geographic strains of pathogens and host, and focuses on studying phenoloxidase (PO) and other enzymes activities in hemolymph, midgut and silk glands. PO activity increased in silkworms infected with Bacillus spp. geographic strain of $B s E$, and $B s C$. The activities of PO and carboxylesterase (CE) in the midgut of infected silkworms gradually increased in fourth larval instars, while it decreased in fifth larval instars. Alkaline phosphatase (ALKP) activity was present on the inner and outer membranes of the silk gland of diseased silkworms. The outer membrane of the silk gland was dyed with Diaminoben-zidine (DAB), but the inner membrane excluded dye. A mixture of the Nitro-Blue-Tetrazolium/ 5-bromo-4-chloro-3indolyl-phosphate (NBT/BCIP) and DAB methods was used to treat the fat body, and both reactions could be detected. Current research into the PO cascade, CE, and ALKP is further reviewed and discussed in detail.
\end{abstract}


Keywords: flacherie; Bombyx mori; enzymes; silk gland; pathology; insect immunity

\section{Introduction}

Flacherie is the most common silkworm disease always broke out in tropic and subtropic area of silkworm rearing, and it inflicts severe damage to the sericulture ever year. The cause of disease is mainly due to the pathogens flacherie virus (FV) or Bacillus spp., which are often confused with other pathogens. Earlier studies have suggested that flacherie disease could also be caused by mixed pathogens, such as cytoplasmic polyhedrosis (CPV), pebrine, or FV. In view of these points, the bacterial disease formerly called flacherie is complex and is classified into different branches, namely: bacterial flacherie, septicemia, and sotto (Ganga et al.,1991). The hemolymph of a diseased silkworm contains a high percentage of adipohemocytes, a low content of proteins, calcium, magnesium, and chloride, and the acidity of the hemolymph also decreases, and the $\mathrm{pH}$ reaches 7.0 (Aruga, 1994). However, it is not clear the interaction between the bacterial and host, especially biochemical changes in the tissues and hemolymph when silkworms were infected by Bacillus spp.

Once microorganisms invade silkworms, the prophenoloxidase (PPO, E.C.1.14.18.1) activating system switches on immediately to promote nonself recognition and respond in terms of melanin production and immunorecognition (Rtcliffe et al., 1984; Leonard et al. 1985; Xia et al., 2018). Experiments suggest that some chemicals such as lipopolysaccharides, lipoteichoic acid, and peptidoglycans from bacterial cell walls can activate a PPO cascade and the antibacterial synthesis system of insects (Rao et al., 2010; Shu et al., 2016). Different and spatial regulation of proPO-activating enzyme (PPAE) and proPOs (PPO) primes the system for innate immunity and cuticular melanization through different pathways and regulation systems (Zou et al., 2015; Lee et al., 2015). PO transforms tyrosine into L-dopa by hydroxylation, which can be oxidized to dopachrome and triggers the cascade to form a package to eliminate pathogenic bacteria (Whitten and Coates, 2017).

$\mathrm{CE}$ is in a superfamily containing the $\alpha / \beta$ hydrolase fold protein domain, which is found in a number of functionally different enzymes that are capable of hydrolyzing a wide range of substrates, including proteases, lipases, esterases, dehalogenases, peroxidases, and epoxide hydrolases. In Bombyx mori, CE consists of two identical subunits that held by non-covalent bonds with a high content of active sites in hydrophobic amino acid residues (Murthy et al., 1996). 87 CE genes were found in B. mori, but only a few genes are responsible for 
organophosphate resistance; the others play important roles in pheromone degradation, neurogenesis, and development regulation and have tissue-specific distribution in the midgut, integument, head, and silk gland (Yu et al., 2009; Kawamoto et al., 2019). However, those mainly involved in detoxification of organophosphate insecticide CEs are mediated nonspecifically (Cui et al., 2011; 2015). CEs also have a close relationship with the synthesis of antibacterial peptides (AMPs) in the fat body and hemocytes in silkworm (Shiotsuki et al., 1999; Ma et al, 2019).

Alkaline phosphatase (ALKP, E.C.3.1.3.1) has long been studied in many creatures such as microorganisms, mammals, and insects. It is well known to be a membrane-bound protein anchored (Low et al., 1986; Bublitz et al., 1993). Two forms of alkaline phosphatase in the midgut of the silkworm Bombyx mori were separated from midgut epithelia (Enguchi et al., 1972). The isoforms of ALKP were named the membrane-bound (mALKP) and soluble ALKP (sALKP) forms (Enguchi et al., 1990). Bacterial infection with Bacillus thuringiensis causes direct damage to the midgut tissue of the silkworm, accompanied by a rapid decline in ALKP activity (Miao, 2002).

Peroxidases (PODs, EC 1.11.1.7) are hemoprotenis containing oxidoreductases with four highly conserved disulfide bridges and approximately $21 \%$ glycosylation (Grey et al., 1998), and catalyzing the oxidation by hydrogen peroxide of a number of substrates such as ascorbate, ferrocyanide, cytochrome $\mathrm{C}$ and the leuco form of many dyes. It is now widely found in plant and animals, for example, glutathione peroxidase and thioredoxin peroxidase in silkworms, and could be detected by horseradish peroxidase (HRP) conjugate antibody. HRP was used as a component of clinical diagnostic kits and for immunoassays (Azevedo et al., 2003; Patmawati et al., 2019). They promote the oxidation of various compounds using naturally occurring peroxides, especially hydrogen peroxide, which are reduced, forming water. In oligophagous insect silkworm, PODs presence both in vesicular structures inside the cytoplasm and in the space between two adjacent absorptive cells, indicating the occurrence of both a transcellular and paracellular permeation route. The peroxidase could be detected from lumen-to-haemolymph and localized in the junctions between adjacent cells. Transcytosis is a key mechanism for proteins absorption in B. mori midgut (Casartelli et al., 2007).

The purpose of this study aimed to comparatively explore biochemical changes, such as the activities of $\mathrm{PO}$ and $\mathrm{CE}$ in the hemolymph and midgut of silkworms infected with different geographic strains of Bacillus spp. The ALKP location in pathological tissue was further 
confirmed by histochemistry in silkworm, and HRP was located in the fat body of the test silkworm as well. It is essential for further exploring of insect biochemical process and application in sericulture.

The flacherie pathogens in the paper refer to Bacillus spp. geographic strains only.

\section{Materials and Methods}

\section{Silkworm Rearing and Infection}

\section{Isolation of the Flacherie}

The pathogen of $B S C$ and $B s E$ were kept in the Laboratory of Insect Physiology and Biochemistry, College of Animal Science, South China Agricultural University. The pathogens were incubated in LB liquid medium to the desired concentration.

The silkworm variety 932 at the fourth larval instar was reared in labeled containers. The silkworms were fed on leaves contaminated with $B s C$ or $B s E$ at $1 \times 10^{8} \mathrm{CFU} / \mathrm{mL}$ in $\mathrm{LB}$ medium to make sure each silkworm intakes $1 \times 10^{4} \mathrm{CFU}$. Sterilized LB medium was used as a control. Thereafter, the silkworms were reared on high-quality mulberry leaves. All experiments were repeated three times.

\section{Collection of Hemolymph Samples}

The bacterial at the concentration of $1 \times 10^{8} \mathrm{CFU} / \mathrm{mL}$ in $\mathrm{LB}$ medium were sprayed on mulberry leaves. In order to prevent from additional bacterial polluting the mulberry, sterilized LB medium was sprayed on mulberry leaves as control. The worms were reared at room temperature $\left(25^{\circ} \mathrm{C}\right.$, Relative Humidity $\left.75-85 \%\right)$. Hemolymph samples' collection began on the second day after infectious mulberry leaves were given. The caudal legs or caudal horns of three diseased silkworms were cut with shears, and hemolymph were collected with eppendrof tubes on ice-bath every other day except the stage of molting. All samples were labeled and kept at $-20^{\circ} \mathrm{C}$ immediately after collection. The hemolymph samples were frozen and thawed only once during the experiment and process of analysis as described subsequently treatment.

\section{Midgut Sample Collection}

The midguts of three diseased silkworms were dissected and collected as a sample, while the midguts of three healthy silkworms were designed as control. The undigested mulberry 
leaves were carefully picked out, and the midguts were washed with $0.01 \mathrm{~mol} / \mathrm{L}$ of PBS, $\mathrm{pH}$ 9.4 to maintain the basic conditions of the silkworm midguts tissues. The tissues of midguts were dried with absorbent paper at room temperature, and weighted with a balance (Sartorius BS323S, Germany) and recorded. The midguts were homogenized in $500 \mu \mathrm{L}$ of SSC buffer $(150 \mathrm{mmol} / \mathrm{L} \mathrm{NaCl}$ and $15 \mathrm{mmol} / \mathrm{L}$ sodium citrate, $\mathrm{pH} 7.0$. Roche) with a sterilized, ceramic grinder and then centrifuged at $6,000 \mathrm{~g}$ for $10 \mathrm{~min}$ at $4^{\circ} \mathrm{C}$. The supernatants from these samples were then used as original enzyme materials.

\section{In situ Hybridization with ALKP and Peroxidase}

The silk gland and fat body were dissected under $4{ }^{\circ} \mathrm{C}$ to keep the activity of enzymes for in situ histochemical reactions. To maintain the activity of ALKP and peroxidase, the following procedures were carried out at $4^{\circ} \mathrm{C}$. Following Harlow and Lane (2006) and the guidance of the manufacturer's instructions of a KD-1508A-VI Microtome (Kedi Instrument and Equipment Co. Ltd., Zhejiang, China), the samples were washed with a solution of $0.1 \%$ phenylhydrazine hydrochloride in PBS, $\mathrm{pH} 7.0$, and cut in to small blocks of tissue approximately $1 \mathrm{~cm}^{2} \times 0.4 \mathrm{~cm}$ in size, followed by chilling in paraffin-embedded tissue blocks on ice before sectioning. All samples were carefully fixed in precooled Bouin's fix fluid solution $(75 \mathrm{~mL}$ of picric acid in saturated aqueous solution, $25 \mathrm{~mL}$ of $40 \%$ formalin aqueous solution, and $5 \mathrm{~mL}$ of acetic acid) overnight at $4^{\circ} \mathrm{C}$. The blocks were trimmed to expose the tissue surface and sectioned at a thickness of about $5 \mu \mathrm{m}$. Other parameters of the microtome were the knife temperature was $-2^{\circ} \mathrm{C}$, and the table temperature was $-30^{\circ} \mathrm{C}$. Using tweezers, ribbons of sections were picked up and floated on the surface of water in a water bath to flatten them out. Tweezers were used to separate the sections, and microscope slides were used to pick the sections out of the water bath, which were then stored upright in a slide rack. Slides were rehydrated by passing the sections through graded ethanol at the concentration of $100 \%, 95 \%, 80 \%$, and $70 \%$ for 3 min each with two changes. Samples were then rinsed in a xylene solution with three changes for $3 \mathrm{~min}$ and finally rinsed in distilled water. The samples were prepared for the next steps of in situ hybridization with NBT and the ALKP substrate BCIP. The chromogen for peroxidase was DAB. ALKP hydrolyzes BCIP to 5-bromo-4-chloro-3-indolyl (BCI) and inorganic phosphate. The BCI, in turn, is oxidized by NBT to form an insoluble dark blue diformazan precipitate after reduction. Therefore, NBT/BCIP and DAB were the best choice for in situ hybridization with ALKP and peroxidase.

\section{Analysis of Enzymes Activity}




\section{The Extraction of Phenoloxidase (PO) from Hemolymph Lysate}

A total of $200 \mu \mathrm{L}$ of hemolymph was mixed with an equal volume of ice-cold AC-buffer (62 $\mathrm{mmol} / \mathrm{L} \mathrm{NaCl}, 100 \mathrm{mmol} / \mathrm{L}$ glucose, $10 \mathrm{mmol} / \mathrm{L}$ EDTA, $30 \mathrm{mmol} / \mathrm{L}$ trisodium citric dehydrates and citric acid monohydrate, $\mathrm{pH}$ 6.8, autoclaved) and washed several times. The mixed solution were then spun at $4,000 \mathrm{~g}$ for $10 \mathrm{~min}$ at $4{ }^{\circ} \mathrm{C}$ in a refrigerated centrifuge (Centrifuge 5810R, Eppendorf,Germany). The resultant cell pellet was resuspended in CACbuffer ( $5 \mathrm{mmol} / \mathrm{L} \mathrm{CaCl}_{2}, 0.01 \mathrm{mmol} / \mathrm{L}$ sodium cacodylate (Sigma), $\mathrm{pH} 7.0$, autoclaved), homogenized with a pre-cold, glass homogenizer, and centrifuged as above. Additionally, cell pellets were lysed and dissolved in $200 \mu \mathrm{L}$ of test solution $(40 \mathrm{~mm} \mathrm{NaOH} ; 145 \mathrm{mmol} / \mathrm{L}$ $\mathrm{NaCl} ; 17 \mathrm{mmol} / \mathrm{L}$ EDTA; $41 \mathrm{mmol} / \mathrm{L}$ citric acid; 5\% of sucrose; $10 \mathrm{mmol} / \mathrm{L}$ guanidine hydrochloride, $\mathrm{pH}$ 7.2, autoclaved). All Eppendorf tubes were placed in an ice bath and then assayed for PO activity.

\section{Assay of PO Activity}

A spectrophotometer (Shimadazu UV-1800, Kyoto, Japan) was used to assay PO activities, using the methodology from Michael (2001). A total of $200 \mu \mathrm{L}$ of test solution was mixed with an equal volume of ice-cold substrate solution (to test PO activity, 1.0 unit of trypsin at 1:250, SABC, was prepared before use); $7 \mathrm{mmol} / \mathrm{L}$ L-dopa (Sigma) in CAC-buffer) and spun at $6,000 \mathrm{~g}$ for $10 \mathrm{~min}$ at $4^{\circ} \mathrm{C}$. The final volumes were adjusted to $2 \mathrm{~mL}$. The units of PO were defined as $1 \mu \mathrm{L}$ of homogenized hemocytes supernatant in test solution $\left(\mathrm{pH} 7.0,30^{\circ} \mathrm{C}\right)$ causing a 0.1 absorbance value change at $490 \mathrm{~nm}$ in 1 min defined as 1 unit of enzyme activity. The empty test solution with substrate solution was used as blank to adjust zero of spectrophotometer.

\section{The Activities of CE and PO from the Midgut}

The methodology used to assay the activity for CE was according to Miao (2002) with a few modifications. The supernatants of the homogenized hemolcytes or midgut tissues were collected and used as original samples. A total of $100 \mathrm{~mL}$ of incubation buffer $(0.1 \mathrm{mmol} / \mathrm{L}$ Tris, pH 8.8; $230 \mu \mathrm{L}$ of $10 \% \mathrm{MgCl}_{2} ; 2 \mathrm{~g} \mathrm{NaCl} ; 100 \mathrm{mg} \alpha$-naphtylacetic acid and $100 \mathrm{mg}$ fast blue R.R salt dissolved in $10 \mathrm{~mL}$ of acetone, and $0.5 \mathrm{~g}$ polyvinylpyrrolidone, Sigma, dissolved in $20 \mathrm{~mL}$ of ethanol and then mixed and left to standstill for $24 \mathrm{~h}$, with the supernatant being used as an incubation buffer) should be prepared before use. A total of 100 $\mu \mathrm{L}$ of the sample was added to $900 \mu \mathrm{L}$ of incubation buffer and incubated at $25^{\circ} \mathrm{C}$ for $25 \mathrm{~min}$. A total of $20 \mu \mathrm{L}$ of EDTA was added to end the reactions. The values of absorbance at 410 
$\mathrm{nm}$ were measured with a spectrophotometer. The $\mathrm{CE}$ activity was defined as $1 \mu \mathrm{L}$ of the original sample causing an absorbance value change of 0.1 in $1 \mathrm{~min}\left(\mathrm{pH} 7.0,25^{\circ} \mathrm{C}\right)$ at $410 \mathrm{~nm}$ being 1 unit of enzyme activity. The incubation buffer was used as blank to adjust zero of spectrophotometer.

The activities of PO were measured as described before.

\section{In Situ Hybridization with ALKP and Peroxidase}

A work solution of NBT/BCIP (Sigma-Aldrich, B3679) and DAB (Sigma-Aldrich, D7679) was diluted accordingly to the manufacturer's recommendations. The sections were observed, and photos were taken using a CX-41 phase-contrast microscope $(\times 1000$, Olympus America Inc., Melville, NY, USA).

\section{Results}

\section{The Activities of PO}

The results of experiments showed that the PO activity increased steadily when silkworms were infected with $B s E$, while the activity was irregular when silkworms were infected with $B s C$; however, the PO activities at the same time among three samples varied significantly difference except the fourth interval (Fig.1). Statistical analysis also showed that there is significantly difference between the groups of hemocyte lysates from control silkworm and those infected with $B s E$ and $B s C$ at $P<0.01$. However, after 18 days of infection, there was more than $81 \%$ death in the silkworms that were infected with $B s C$, while less than $15 \%$ died when infected with $B s E$. The toxicity is largely due to the laboratory purified and incubated $B S C$ which might contain more toxic components in the bacterial wall. On the other hand, when we compared columns at the $2^{\text {nd }}$ day and $6^{\text {th }}$ day, it was clearly that the silkworms which recovered from the infectious disease showed higher PO activities than those of uninfected silkworms. 


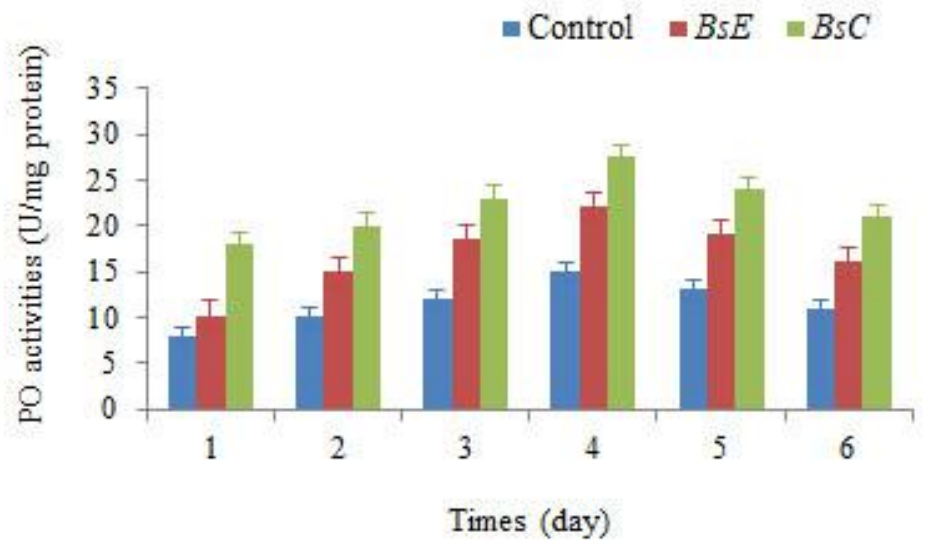

Fig. 1 The PO activities of hemocyte lysates from Bombyx mori infected with different geographic strains of flacherie.

Control, $B s E$ and $B s C$ indicate that of silkworms fed on infectious mulberry leaves with LB medium and different geographic strains of Bacillus spp, respectively. The error bars indicated standard deviation errors of the data. SPSS statistical analysis revealed that there is significant difference at the level of $0.01(P<0.01)$ between days of control and infected silkworm with $B s E, B s C$. Paried $t$ test showed significant difference at 0.01 level $(P<0.01)$ between all groups.

\section{Activities of PO and CE in the Midgut}

Flacherie disease in silkworms mainly causes biochemical changes in the midgut and affects nutrition transport into the hemocoel. PO activity in the midgut of infected silkworms gradually increases in the fourth instar for 3 days and then gradually decreases in the newly molted fifth instar. A peak in the PO level was observed in the fifth instar but the concentration of PO was much lower than that in the fourth instar. According to statistical analysis, there is significant difference between the groups of silkworm infected with $B s E$ at the level of $0.01(P<0.01)$; the other two groups exist significant differences at $P<0.05$. There is significant difference between the samples from the same day at $P<0.01$ (Fig.2). These results suggested that bacteria activate the PO cascade at the beginning of infection, which causes a high level of PO in the fourth instar, which then decreases in the fifth instar. Further, the PO activity of the midgut collected from infected silkworms was higher than that of control silkworms. 


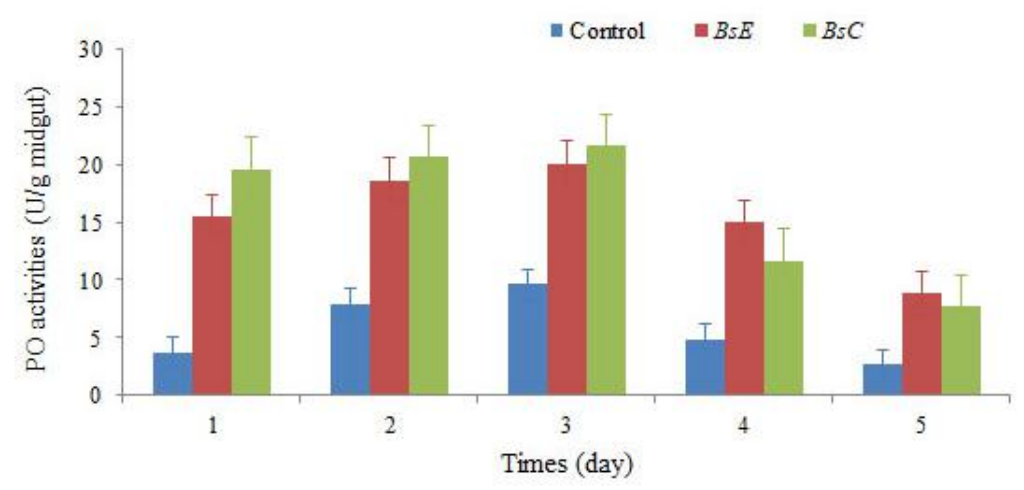

Fig. 2 PO Activities of the midgut from silkworm, Bombyx mori, infected with different geographic strains of flacherie.

$B s C, B s E$, and control indicate the PO activities in the midgut of infected silkworms fed with mulberry leaves with different geographic strains of Bacillus spp, and LB medium as control, respectively. The $\mathrm{X}$-axis represents the days of development of silkworms after infection with Bacillus spp or control from the $4^{\text {th }}$ to $5^{\text {th }}$ larval instar. The error bars indicated standard deviation errors of the data. SPSS statistical analysis revealed that there is significant difference at the level of $0.01(P<0.01)$ between the days of infected silkworm with $B S E$ but no significant between the days of control and those silkworms infected with $B s C$; paired $t$ test showed that significant difference at $P<0.01$ between the groups of control and infected silkworm with $B S C$ or $B s E$, but no significant difference between silkworms infected with $B s C$ and $B s E$.

The activity of $\mathrm{CE}$ in the hemolymph of the infected silkworm gradually increased during the fourth instar and the period after molting but decreased on the tenth day in the fifth instar. Infected silkworms showed a lower level of CE activity than that of controls, but the activity increased to a small plateau on the eighth day after infection (Fig.3). Statistical analysis revealed that significant differences between the samples of $B s C$ infected silkworms and control at the level of $0.05(P<0.05)$; those samples extracted from silkworms infected with $B s E$ and control at the level of $0.01(P<0.01)$ after infection 6 to 7 days. There are significant differences in all test three groups of samples at the level of $0.01 \quad(P<0.01)$. The related changes in PO and CE activity from the fourth instar and fifth larval instar suggested that the biochemical changes in the midgut of infected silkworms were consistent in the defense system. 


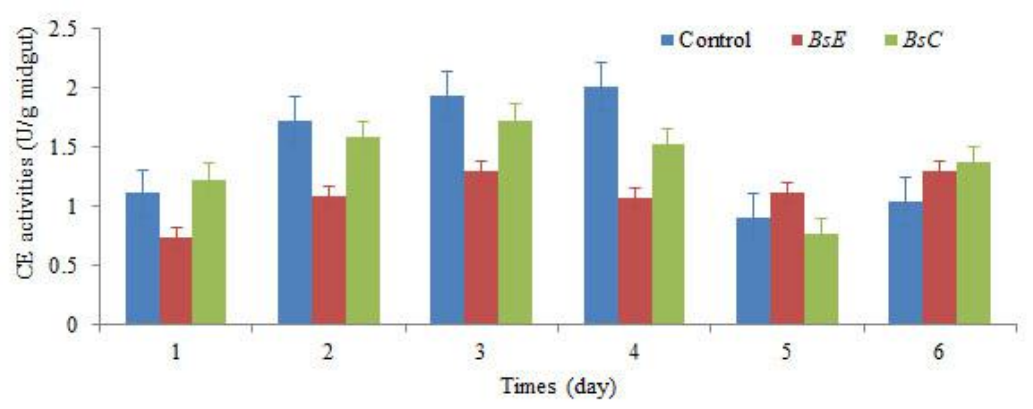

Fig. $3 \mathrm{CE}$ activities of the midgut from the silkworm Bombyx mori infected with different geographic strains of flacherie.

$B s C, B s E$, and control indicate the $\mathrm{CE}$ activities in the midgut of infected silkworms fed mulberry leaves with different geographic strains of Bacillus spp, and LB medium, respectively. The $\mathrm{X}$-axis represents the days of development of silkworms after infection with Bacillus spp or control from the $4^{\text {th }}$ to $5^{\text {th }}$ instar. The $\mathrm{Y}$-axis represents the CE activities (U) per gram of midgut. The error bars indicated standard deviation errors of the data. SPSS statistical analysis revealed that there are significant differences between days at the level of $0.01(P<0.01)$ in all group samples, but paired $t$ test didn't show significant differences between all groups.

\section{In Situ Histochemical Staining of ALKP and Peroxidase}

In order to study ALKP and peroxidase activities in the tissues of the silk gland and fat body and confirm the location of mALKP and sALKP, the target tissues were anatomized by microtome. The tissue sections were cut at $-2^{\circ} \mathrm{C}$, while the table temperature was maintained at $-30^{\circ} \mathrm{C}$ to protect enzyme activities. The inner and outer membranes of the silk gland were dyed with NBT/BCIP suggesting that the activity of ALKP was very high in the membrane of silk glands from diseased silkworms (Fig. 4-1).
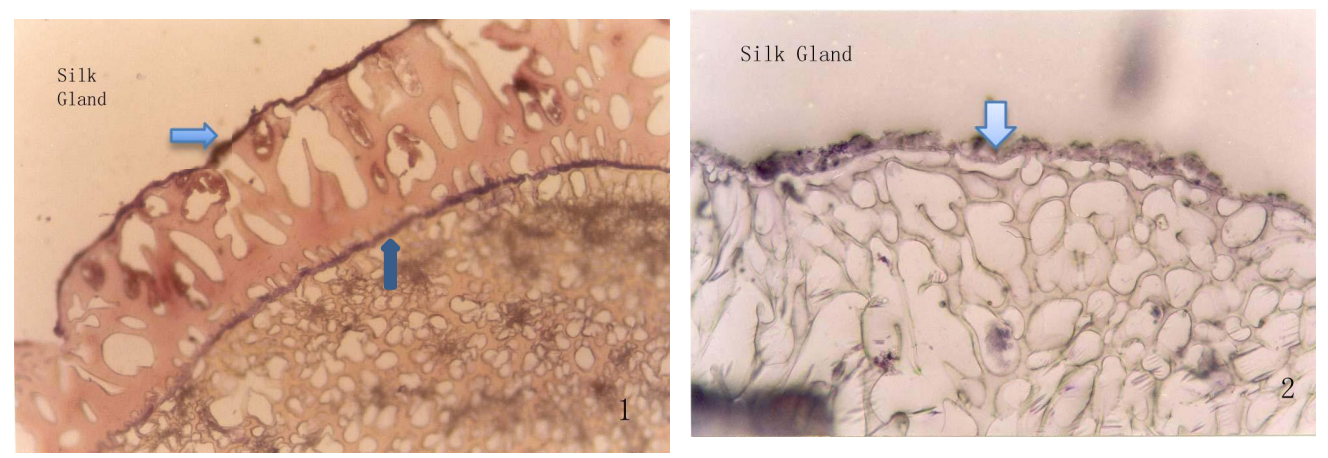

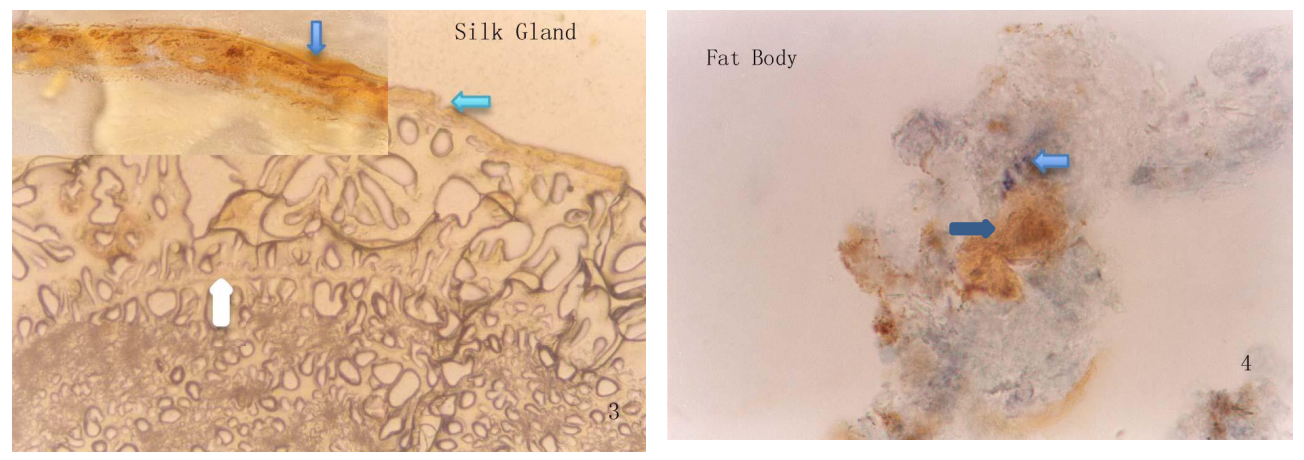

Fig. 4 Histochemical staining with NBT/BCIP or DAB method in the silk gland and fat body of diseased and healthy silkworms.

Fig. 4-1 (100×) shows the silk gland from a diseased silkworm. Blue arrows indicate mALKP located on the inner and outer membranes of silk gland which were dyed with NBT/BCIP, while Fig. 4-2 (400×) (control) indicates little staining under the same conditions in healthy silkworm (azure arrow); Fig. 4-3 (100×): The outer membrane was dyed with the DAB method, but the inner membrane excluded the dye (blue and white arrows) from a diseased silkworm. The window on the upper left displays an enlarged outer membrane of the silk gland (1000×). Fig. 4-4 (400×) shows mixed NBT/BCIP and DAB staining of fat body from healthy silkworm.

The inner membrane of healthy silkworm was dyed with azure (light blue arrow indicated in Fig. 4-2) under the same conditions. The most interesting result was that the inner membrane of the silk gland did not have strong staining with the DAB method, while the outer membrane did (Fig. 4-3). In the fat body, both NBT/BCIP and DAB reacted with their own substrates and stained this tissue (Fig. 4-4) indicating that the fat body is an important tissue of ALKP and peroxidase cascades.

\section{Discussion}

Infectious diseases of silkworms cause major economic loss to the silk industry, especially in terms of silkworm breeding and cocoons production. From the 1980s, professional and comprehensive prevention measures have become common to keep silkworms free of infectious diseases.

Our results showed that PO and CE activities of the hemolymph and midguts from diseased silkworms increased when silkworms were infected with $B s E$ or $B s C$. PO activity in the 
midgut of infected silkworms gradually increased in the fourth larval instar (3 days) and newly molted silkworms in the fifth larval instar. PO levels were high in the middle of the instar and then gradually decreased.

Pathogens cause humoral and cellular immunity responses at the beginning of infection. Humoral immunity includes lysozymes, antibacterial peptides (cecropins, proline, and glycine-rich peptides), defensins, and antifungal peptides. Three kinds of PO (granular, wound, and hemolymph) and a lactase-type PO are present in insects. It was reported that the zymogen of PO is activated through the action of a serine protease cascade, and the oenocytoid hemocytes of Bombyx mori are the major site of PO synthesis (Ashida et al. 1998). Therefore, the level of PO activity directly influences the proliferation of oenocytoid hemocytes in the cavity of infected silkworms.. In the midgut of a silkworm, the issues might be more complicated because of the resident gut microbiota and the physical barrier of the peritrophic membrane. In the hindgut, PPO is secreted to induce melanization of the feces for cleaning fecal microorganisms (Shao et al., 2012), but it is secreted into the foregut and activated by a chymotrypsin-like enzyme to catalyze plant phenolics into intermediates in the diet (Wu et al., 2015). An increasing number of works have revealed that many factors are involved in the PPAE cascade in silkworms. Serine proteases and serine protease homologs of Bombyx mori (BmSPs, BmSPHs) are large families that are involved in the PPAE cascade leading to the final events of immunity and melanization. However, nearly all enzymes such as BmSP14, BmSP21, BmSPH1, BmSPH2, and even PPAE and PPO themselves are secreted as inactive forms in the plasma and in aggregated hemocytes, where they have an effective invading microorganism-surveillance network but putatively function in active forms at functional sites (Tokura et al., 2014; Shu et al., 2016). These enzymes always act in multiple roles in the development and immunity of silkworms. For example, BmSP1 participates in the regulation of nodule melanization and in larval to pupal transformation (Lee et al., 2018).

In contrast, the activation of PPO and antibacterial peptide pathways is negatively regulated by serine protease inhibitors (Serpins). These extracellular BmSerpins are essential components and play an important regulatory role in the process of silkworm immunity by regulating the serine protease cascade pathway (Zhao et al., 2010; Wang et al., 2018). BmSerpin-3 was detected to be primarily transcribed within the fat body, hemocytes, and epidermis. The results showed that BmSerpin-3 might act as a negative regulator of PPO activation and inhibit the synthesis of antibacterial peptides (Wang et al., 2017). The mRNA abundance of BmSerpin-5 increases dramatically in the fat body after bacterial infection 
(Tenaka, et al., 2008). The silkworm clip domain proteins BmSP6 and SP21 form a complex with BmSerpin-5, which suggests that BmSP6 and SP21 are the cognate proteases of BmSerpin-5 and are essential in the serine protease cascade that activates the Toll and PPO pathways (Li et al., 2016). RT-PCR results showed that BmSerpin-6 was expressed highly in hemocytes, the midgut, and the fat body after challenging silkworm with Micrococcus luteus or Serratia marcescens, but the transcript levels of PPO were reduced significantly in the fat body and hemocytes (Li et al., 2017). Similar experimental data demonstrated the functions of Serpin-14 in Antheraea pernyi (Kausar et al., 2017) and Serpin-15 in Bombyx mori (Liu et al., 2015). These data also demonstrated that Serpin-32 has significant inhibitory activity against trypsin and delayed or inhibited the melanization of hemolymph by inhibiting the activation of prophenoloxidase in larval hemolymph (Wang et al., 2019).

Furthermore, peptidoglycan recognition proteins (PGRPs) can specifically recognize and bind to highly conserved pathogen-associated molecular patterns (Shu et al., 2016). Zhang et al. (2019) cloned and expressed five short types of PGRPs (PGRP-S) from silkworm. These showed significant agglutination activity toward positive and negative bacteria, and most notable amidase activity toward Bacillus spp. meso-diamnopimelic acid peptidoglycan (DAP-PGN) is also capable of activating the imd pathway. Recent studies have also suggested that DAP decarboxylase catalyzes the final step in the de novo L-lysine biosynthetic pathway (Son and Kim, 2018). DAP or Lys PG, PGRP-S1, and Serpin-14 together trigger the PPO activation system in a $\mathrm{Ca}^{2+}$-dependent manner (Wang et al., 2017). PGRP-S1 binds to bacterial PGN to activate the PPO pathway to produce melanin and encapsulate the pathogen. In the silkworm, PGRP-S5 acts as a sensor, a modulator, and an effector in the humoral immune system (Chen et al., 2014) as well as functioning as a pattern recognition receptor (PRR) to bind Lys- and DAP-PGN from certain bacterial strains and then activates the PO pathway in the same manner (Wang et al., 2019). Recently, homologs of the QM gene, a novel gene that was first isolated as a putative tumor suppressor gene from the Wilms; tumor cell line, was cloned from Bombyx mori (Zhou et al., 2019). BmQM mRNA and protein were highly expressed in the silk gland and fat body and expressed in all stages of silkworm growth. It affects some immune gene expression and negatively regulates PPAE.

Infectious disease caused the activities of CEs to gradually increase in the fourth larval instar and the period after molting, but this decreased on the tenth day in the fifth larval instar. Infected silkworms showed a lower level of CE activity than controls did, but the activity 
increased to a small summit on the eighth day. The CE activity peak was present at a time that coincided with cecropins, antibacterial peptides that are synthesized and induced by $E$. coli $\mathrm{K}_{12} \mathrm{D}_{31}$ in Antherea pernyi (Qu et al., 1982) and Bombyx mori (Sumida et al., 1992). The activity of CE in the midgut is largely dependent on silkworms being in the stage of molting and the state of feeding, the qualities of mulberry leaves, and temperature if the silkworms are not reared on insecticide polluted leaves. CE activity also changes in the different species of silkworms and sexes (Miao et al., 2002). The characters of the CE superfamily are somewhat an exceptional example of enzyme activity because some of these enzymes are strongly substrate selective. For example, consider juvenile hormone esterase and acetylcholinesterase, whereas others are less specific (esterases) or non-catalytic proteins, such as neuroligins, gliotactins, and neurotactins. CEs are widely distributed in nature. Some metabolic CEs belong to the odorant-degrading enzyme family in silkworm and play a key role in the degradation of acetate sex pheromones and host plant volatiles (Ishida and Lee, 2005). However, those mainly involved in detoxification of organophosphate insecticide CEs are mediated nonspecifically (Cui et al., 2011; 2015). Feeding on phoxim (Gu et al., 2015) and chlorantraniliprole (Mao et al., 2019), CE activities in the fat body of silkworm are upregulated by $24 \mathrm{~h}$, and then they are decreased through regulation of the key genes in the $\mathrm{PI} 3 \mathrm{~K} / \mathrm{Akt} / \mathrm{CncC}$ signaling pathway at the mRNA level. RNAi experiments showed that several potential transcriptional regulatory elements such as $C r o c$ and $D f d$ were essential for basal and rutin-induced transcriptional activity. The activity of the CE-10 promoter was highest in the Malpighian tubule, followed by the fat body, silk gland, midgut, epidermis, and hemocytes (Zhao et al., 2014).

Giving the resistance of silkworm species to densonucleosis virus (BmDNV, Gao et al., 2007), nuclear polyhedrosis virus (BmNPV, Bao et al., 2009), and cytoplasmic polyhedrosis virus (BmCPV, Wu et al., 2013), the activities of CEs varied in the subsequent examination. Recent studies have demonstrated that lipase from intestinal bacterium, in particular the lipase-producing bacterial strain Bacillus pumilus SW41, is a potential antiviral factor for silkworm against BmNPV (Liu et al., 2018). CEs and other protease-like hemocyte proteases, antitrypsin, and aminopeptidase $\mathrm{N}$ are higher in resistant silkworms compared to susceptible individuals (Guo et al., 2016). Several SPs and Serpins are up-regulated after infection indicating that viruses activate PPAE cascades in these species (Zhang et al., 2015; Lü et al., 2018). In contrast, although results showed that the extent of melanization was significantly higher in a susceptible strain relative to that of a resistant strain, and a majority of Serpins 
were up-regulated in a resistant strain, further experiments demonstrated that Serpin-5, Serpin-9, and Serpin-19 reduced PO activity indicating that a resistant strain inhibited viral infection independent of the melanization pathway (Chen et al., 2019).

CEs also have a close relationship with the synthesis of antibacterial peptides (AMPs) in the fat body and hemocytes in silkworm. Some homologs of silkworm CEs are bacterially inducible and are stimulated by lipopolysaccharides but clearly differ from non-inducible CEs (Shiotsuki et al., 1999). Albert et al. (2011) reported that a novel leucine-rich repeat protein behaves as a typical secreted immune protein. It is expressed simultaneously with carboxylesterase upon treatment with bacteria or other elicitors of immune response. During bacterial invasion, pathogens produce toxins and other virulence factors to counteract host immune responses. In these processes, the pathways leading to PPO activation and AMPs synthesis in the silkworm are affected by metalloproteases (Ma et al, 2019).

Biogenic amines such as octopamine and dopamine are always present in the nervous systems of insects. Octopamine is well known as a typical phenolic biogenic amine acting as a neurotransmitter, neuromodulator, and neurohormone. The other amine, dopamine is also a basic component of the PPO catalysis cascade in the melanization process. Thus, a chemiluminescent reporter gene assay system that detects secreted placental alkaline phosphatase (ALKP) transcriptionally regulated by the cAMP response element for a silkworm octopamine receptor might be useful in high-throughput screening to develop octopamine receptor-specific insecticides (Hiroto et al., 2012). ALKP activity also used as an indicator of quality control, hazard analysis, and critical control points for the industrialization of edible insects (Grabowski et al., 2018).

ALKP catalyzes every kind of alcohol and phenol and transfers phosphate that is localized on the cell membrane for active transportation. This was consistent with the experiments conducted here, where active ALKP was present on the inner and outer membrane of the silk glands of diseased silkworms compared to healthy silkworms. This finding suggested that ALKP plays a key role in disease resistance. The last enzyme was cytochrome oxidase in the electronic transfer system on the inner mitochondrial membrane. DAB itself was oxidized by the deoxidizing action of cytochrome $\mathrm{C}$, and the later was oxidized again by cytochrome $\mathrm{A}$. The reaction process resulted in DAB continually being oxidized and the product of the reaction precipitating on the reactive part of the tissue. DAB was detected (Fig. 4-4) in the fat body. Fig. 4-3 demonstrates that this reaction only occurred on the outer membrane of silk glands in diseased silkworms, and no cytochrome $\mathrm{C}$ and hydrogen peroxide could be found 
on the inner membrane.

In situ histochemical studies suggested that mALKP in the membrane of silk glands was active in diseased silkworms, where only the outer membrane was dyed by the DAB method. The reaction only proceeded on the outer membrane of silk glands in diseased silkworms, and there was no incidence of cytochrome $\mathrm{C}$ or hydrogen peroxide staining on the inner membrane. As a control, a mixed NBT/BCIP/DAB method stained the fat body, showing that both reactions could be detected.

Another enzyme which also involved in defense against pathogens is PODS. It converts colorless or non-fluorescent aromatic substrates into colored and/or fluorescent molecules in the presence of hydrogen peroxide so as to be used in clinical diagnostics kits based on enzyme-linked immunosorbent assay (ELISA). In insects, PODS function in hardening of the chorion (Li et al., 1995), cuticular sclerotization (Andersen, 2010), innate immunity (Choudhury et al., 2019), resistance to NPV infection (Zhang et al., 2015), and against oxidative damage (Corona et al., 2006). It was found that PODS crossed the intestinal epithelial barrier in a time-dependent manner and located at the midgut epithelium of silkworm (Casartell et al., 2011). However, it was not clear whether it is exist in important detoxication tissue of insect, i.e. fat body. The histochemical studies suggested the question of how the fat body functioning in silkworm, nearly involved all physiology and biochemistry processes of reactions.

\section{Summary}

Taken together, the PO activities of hemolymph were different when silkworms were infected with geographically different Bacillus spp strains. The activities of PO and CE in the midgut of infected silkworms varied by stage, but the characteristics of the CE superfamily are somewhat exceptional in that they are non-specific enzymes in terms of substrate selectivity. ALKP was actively present on the inner and outer membrane of the silk gland of diseased silkworms. The outer membrane of the silk gland was stained by DAB, but the inner membrane excluded stain. Mixed NBT/BCIP and DAB treatment stained the fat body, showing that both reactions indicated active ALKP and peroxidase enzymes.

In other words, according to our results, infection stimulates the serpin cascade related to PPO activity, synthesizing antibacterial substances and detoxifying the toxic products of bacterial metabolism and host defenses. 


\section{Funding:}

This study was kindly supported by a grant from the Department of Guangdong Science and Technology (No.2017A020225032) and Guangdong Provincial Sericultural Research and Engineering Center.

\section{References}

[1] Albert Š, Gätschenberger H, Azzami K, Gimple O, Grimmer G, Sumner S, Fujiyuki T, Tautz J, Mueller MJ. (2011) Evidence of a novel immune responsive protein in the Hymenoptera. Insect Biochem Mol Biol. 41: 968-981. Doi:10.1016/j.ibmb. 2011.09.006.

[2] Andersen SO. (2010) Insect cuticular sclerotization: A review. Insect Biochem Mol Biol.,40:166-178. Doi:10.1016/j.ibmb.2009.10.007.

[3] Aruga Hisao. (1994) Principles of Sericulture (Translated from Japanese). Oxford and IBH Publishing Co.Pvt.Ltd.66 Janpath, New Delhi, 110001.

[4] Ashida $M$ and Brey P.T.( 1998) Recent advances in research on the insect prophenoloxidase cascade. In "Molecular Mechanisms of Immune Responses in Insects", 135-172. Edit by Paul T. Brey and Dan Hultmark. Chapman \& Hall, 2-6 Boundary Row, London SE1 8HN.

[5] Ashida M. and Dohke K.(1980) Activation of prophenoloxidase by the activating enzyme of the silkworm, Bombyx mori. Insect Biochem.10: 27-47. Doi:10.1016/0020$1790(80) 90036-0$.

[6] Azevedo AM, Martins VC, Prazeres DMF, Vojinović V, Cabral JMS, Fonseca LP. (2003) Horseradish peroxidase: a valuable tool in biotechnology. Biotechnol.Annu. Rev. 9: 199-247. Doi:10.1016/S1387-2656(03)09003-3.

[7] BaoYY, Tang XD, Lv ZY, Wang XY, Tian CH, XuYP, Zhang CX. (2009) Gene expression profiling of resistant and susceptible Bombyx mori strains reveals nucleopolyhedro- virus-associated variations in host gene transcript levels. Genomics. 94: 138-145. Doi:10.1016/j.ygeno.2009.04.003

[8] Bublitz R., Armesto J., Hoffmann-Blume E., Schulze M., Rhode H., Horn A, Aulwurm S., Hannappel E. and Fischer W. (1993) Heterogeneity of glycosylphosphatidyl- inositolanchored alkaline phosphatase of calf intestine. Eur. J. Biochem. 217: 199- 207. Doi:10.1111/j.1432-1033. 1993.tb18234.x 
[9] Casartelli M, Corti P, Cermenati G, Grimaldi A, Fiandra L, Santo N, Pennacchio F, Giordana B. (2007) Absorption of horseradish peroxidase in Bombyx mori larval midgut. J Insect Physiol.53:517-525. Doi:10.1016/j.jinsphys.2007.02.004.

[10] Chen KK, Liu C, He Y, Jiang HB, Lu ZQ. (2014) A short-type peptidoglycan recognition protein from the silkworm: expression, characterization and involvement in the prophenoloxidase activation pathway. Dev. Comp. Immunol. 4: 1-9. Doi: 10.1016/j.dci.2019.01.015.

[11] Chen TT, Hu N, Tan LR, Xiao Q, Dong ZQ, Chen P, Xu AY, Pan MH, Lu C. (2019) Resistant silkworm strain block viral infection independent of melanization. Pesti Biochem Physiol.154:88-96. Doi:10.1016/j.pestbp.2018.12.012.

[12] Choudhury T P, Lalita G, Kumar S. (2019 ) Identification, characterization and expression analysis of Anopheles stephensi double peroxidase. Acta Tropica. 190: 210219. Doi: 10.1016/j.actatropica.2018.10.008.

[13] Corona M, Robinson GE. (2006) Genes of the antioxidant system of the honey bee: annotation and phylogeny. Insect Mol. Biol. 15 (5): 687-701. Doi:10.1111/j.13652583.2006.00695.x.

[14] Cui F, Lin Z, Wang HS, Liu SL, Chang HJ, Reeck G, Qiao CL, Raymond M, Kang L. (2011) Two single mutations commonly cause qualitative change of nonspecific carboxyl-esterases in insects. Insect Biochem Mol Biol. 41: 1-8. Doi:10.1016/ j.ibmb.2010.09.004

[15] Eguchi M, Azuma M, Yamamoto H, Takeda S. (1990)Genetically defined membranebound and soluble alkaline phosphatases of the silkworm: their discrete localization and properties. Prog Clin Biol Res. 344:267-87.PMID:2392471.

[16] Eguchi M. and Kuriyama K. (1985) Purification and characterization of membranebound alkaline proteases from midgut tissue of the silkworm, Bombyx mori. J. Biochem. 97:1437-1445. Doi: 10.1093/oxfordjournals.jbchem.a135198.

[17] Eguchi M., Sawaki M. and Suzuki Y. (1972) Multiple forms of midgut alkaline phosphatase in the silkworm: separation and comparison of two isoenzymes. Insect Biochem. 2:167-174. Doi:10.1016/0020-1790(72)90050-9.

[18] Ganga G. and J.Sulochana Chetty. (1991).An Introduction to Sericulture. Oxford and IBH Publishing Co. Pvt.Ltd. 66 Janpath, New Delhi, 110001.

[19] Grabowski NT, Olivas JF, Lozano DG, Kehrenberg C, Aguilar DG. (2018) Assessment of pasteurisation of edible insects using enzymatic tests (activity of alkaline phosphatase 
and lactoperoxidase) applied in dairy products. Food Sci Technol Int. 24(8):699-704. Doi: $10.1177 / 1082013218789815$.

[20] Gray JS, Yun Yang B, Montgomery R. (1998) Heterogeneity of glycans at each Nglycosylation site of horseradish peroxidase. Carbohydr Res. 311:61-9. Doi: 10.1016/s0008-6215(98)00209-2.

[21] Gu ZY, Li FC, Wang BB, Xu KZ, Ni M, Zhang H, Shen WD, Li B. (2015) Differentially expressed genes in the fat body of Bombyx mori in response to phoxim insecticide. Pesti Biochem Physiol.117: 47-53. Doi: 10.1016/j.pestbp. 2014.10.007.

[22] Guo HZ, Jiang L, Xia QY. (2016) Selection of reference genes for analysis of stressresponsive genes after challenge with viruses and temperature changes in the silkworm Bombyx mori. Mol. Genet. Genomics..291(2):999-1004. Doi : 10.1007/ s00438-015$1125-4$.

[23] Harlow E and Lane D. (2006) Preparing paraffin tissue sections for immunostaining. CSH Protoc. (1) pii: pdb.prot4329. Doi: 10.1101/pdb.prot4329.

[24] Hiroto O, Hiroshi O, Naotaka H, Tetsuya I, Yoshihisa O, Shigeru M, Kenji K.( 2012) A secreted placental alkaline phosphatase-based reporter assay system for screening of compounds acting at an octopamine receptor stably expressed in a mammalian cell line. Biosci. Biotechnol. Biochem. 76 (1): 209-211. Doi:10.1271/bbb.110690.

[25] Ishida Y and Leal WS. (2005) Rapid inactivation of a moth pheromone. Proc. Natl. Acad.Sci. U. S. A. 102: 14075-14079. Doi:10.1073pnas.0505340102 PN.

[26] Kausar S, Abbas MN, Qian C, Zhu BJ, Sun Y, Sun YX, Wang L, Wei GQ, Maqsood I, Liu CL. (2017 ) Serpin-14 negatively regulates prophenoloxidase activation and expression of antimicrobial peptides in Chinese oak silkworm Antheraea pernyi. Dev Com Immunol. 76: 45-55 Doi:10.1016/j.dci.2017.05.017

[27] Kawamoto M., Jouraku A., Toyodac A., Yokoi K., Minakuchi Y, Katsuma S, Fujiyama A, Kiuchi T, Yamamoto K, Shimada T. (2019) High-quality genome assembly of the silkworm, Bombyx mori. Insect Biochem Mol Biol. 107 :53-62. Doi:10.1016 /j.ibmb.2019.02.002.

[28] Lee KS, Kim BY, Choo YM, Jin BR. (2018) Dual role of the serine protease homolog BmSPH-1 in the development and immunity of the silkworm Bombyx mori. Dev Com Immunol. 85: 170-176. Doi:10.1016/j.dci. 2018.04.011. 
[29] Leonard C. Ratcliffe N. A. and Rowley A. F. (1985) The role of prophenoloxidase actiation in non-self recognition and phagocytosis by insect blood cells. J. Insect Physiol. 31(10): 789-799. Doi: 10.1016/0022-1910(85)90072-1.

[30] Li B, Yu HZ, Ye CJ, Ma Y, Li X, Fan T, Chen FS, Xu JP.( 2017) Bombyx mori Serpin-6 regulates prophenoloxidase activity and the expression of antimicrobial proteins. Gene. 30;610:64-70. Doi: 10.1016/j.gene.2017.02.011.

[31] Li JL, Li M, Zhe L, Zhen Z, Lu ZQ. (2016) Serpin-5 regulates prophenoloxidase activation and antimicrobial peptide pathways in the silkworm, Bombyx mori. Insect Biochem Mol Biol. 73: 27-37. Doi: 10.1016/j.ibmb.2016.04.003

[32] Liu DG, Wang L, Yang L, Qian C, Wei GQ, Dai LS, Li J, Zhu BJ, Liu CL. (2015) Serpin-15 from Bombyx mori inhibits prophenoloxidase activation and expression of antimicrobial peptides. Dev Com Immunol. 51: 22-28 . Doi:10.1016/j.ibmb. 2016.04.003

[33] Low M. G., Ferguson M. A. J., Futerman A. H. And Silman I. (1986) Covalently attached phosphatidylinositol as a hydrophobic anchor for membrane proteins. Trends Biochem. Sci. 11: 212-215. Doi:10.1016/0968-0004(86)90009-5.

[34] Ma L, Zhou LZ, Lin JS, Ji JY, Wang Y, Jiang HB, Shen XH, Lu ZQ. (2019) Manipulation of the silkworm immune system by a metalloprotease from the pathogenic bacterium Pseudomonas aeruginosa. Dev Comp Immunol. 90:176-185. Doi: 10.1016/j.dci.2018.09.017.

[35] Mao TT, Li FC, Fang YL, Wang H, Chen J, Li MX, Lu ZT, Qu JW, Li JX, Hu JH, Cheng XY, Ni M, Li B. (2019) Effects of chlorantraniliprole exposure on detoxification enzyme activities and detoxification-related gene expression in the fat body of the silkworm, Bombyx mori. Ecotoxicol Environ Safety. 176: 58-63. Doi: 10.1016/j.ecoenv.2019.03.074.

[36] Miao YG. (2002) Studies on the activity of the alkaline phosphatase in the midgut of infected silkworm, Bombyx mori L. J. Appl. Ent. 126:138-142. Doi: 10.1046/j.14390418.2002.00625.x

[37] Patmawati, Minamihata K, Tatsuke T, Lee J M, Kusakabe T, Kamiya N. (2019) Functional horseradish peroxidase-streptavidin chimeric proteins prepared using a silkworm-baculovirus expression system for diagnostic purposes. J Biotechnol. 297: 2831. Doi: 10.1016/j.jbiotec.2019.03.007. 
[38] Qu Z, Steiner H, Engström A, Bennich H, Boman HG. (1982) Insect immunity: isolation and structure of cecropins B and D from pupae of the Chinese oak silk moth, Antheraea pernyi. Eur J Biochem. 127(1):219-224. Doi: 10.1111/j.1432-1033. 1982. tb06858.x

[39] Rao XJ, Yu XQ. (2010) Innate immune responses in the Chinese oak silkworm, Antheraea pernyi. Dev Com Immunol. 34: 1119-1128. Doi:10.1016/j.dci.2010.06.007.

[40] Ratcliffe NA, Leonard C, Rowley A.F. (1984) Prophenoloxidase activation: nonself recognition and cell cooperation in insect immunity. Sci. 226(4674): 557-559. PMID: 17821514.

[41] Shao Q, Yang B, Xu Q, Li X, Lu Z,Wang C, Huang Y, Soderhall K, Ling E. (2012) Hindgut innate immunity and regulation of fecal microbiota through melanization in insects. J Biol Chem. 287: 14270-14279. Doi: 10.1074/jbc.M112.354548

[42] Shiotsuki T, Kato Y. (1999,) Induction of carboxylesterase isozymes in Bombyx mori by E. coli infection. Insect Biochem Mol Biol. 29: 731-736. Doi:10.1016/S09651748(99)00054-5

[43] Shu M, Mang DZ, Fu GS, Tanaka S, Endo H, Kikuta S, Sato R. (2016) Mechanisms of nodule-specific melanization in the hemocoel of the silkworm, Bombyx mori. Insect Biochem Mol Biol. 70 :10-23, Doi: 10.1016/j.ibmb.2015.12.005

[44] Son H F, Kim K J. (2018) Structural basis for substrate specificity of mesodiaminopimelic acid decarboxylase from Corynebacterium glutamicum. Biochem Biophysi Res Commun. 495 :1815e1821. Doi:10.1016/j.bbrc.2017.11.097.

[45] Sumida M, Ichimori H, Johchi S, Takaoka A, Yuhki T, Mori H, Matsubara F. (1992) Antibacterial activity inducible in the haemolymph of the silkworm, Bombyx mori, by injection of formalin-treated Escherichia coli K-12 during the fifth larval instar and pharate adult development. Comp Biochem Physiol B.,101(1-2):165-71. Doi: 10.1016/0305-0491(92)90174-P.

[46] Takeda S, Azuma M, ItohM, Eguchi M. (1993) The strain difference and analysis of polymorphic nature of membrane-bound alkaline phosphatase in the midgut epithelium of the silkworm, Bombyx mori. Com Biochem Physiol. Part B: Com Biochem. 104(1): 81-89. Doi:10.1016/0305-0491(93)90341-2

[47] Tanaka H., Ishibashi J., Fujita K., Nakajima Y., Sagisaka A., Tomimoto K., Suzuki N., Yoshiyama M., Kaneko Y., Iwasaki T., Sunagawa T., Yamaji K., Asapka A., Mita K., Yamakawa M., (2008) A genome-wide analysis of genes and gene families involved in 
innate immunity of Bombyx mori. Insect Biochem. Mol. Biol. 38:1087-1110. DoiOI:10.1016/j.ibmb.2008.09.001.

[48] Tokura A, Fu GS, Sakamoto M, Endo H, Tanaka S, Kikuta S, Tabunoki H, Sato R. (2014) Factors functioning in nodule melanization of insects and their mechanisms of accumulation in nodules. $\mathrm{J}$ Insect Physiol. 60: 40-49. Doi:10.1016/j.jinsphys. $\underline{2013.11 .003 .}$.

[49] Wang L, Yang L, Zhou XS, Li TH, Liu CL. (2018) A clip domain serine protease stimulates melanization activation and expression of antimicrobial peptides in the Chinese oak silkworm, Antheraea pernyi. J Asia-Pacific Entomol. 21: 864-871. Doi: 10.1016/j.aspen.2018.06.008.

[50] Wang LL, Liu HW, Fu HY, Zhang L, Guo PC, Xia QY, Zhao P. (2019) Silkworm serpin32 functions as a negative-regulator in prophenoloxidase activation. Dev Com Immunol. 91: 123-131. Doi:10.1016/j.dci.2018.10.006.

[51] Wang Q, Ren MJ, Liu XY, Xia HC, Chen KP. (2019) Peptidoglycan recognition proteins in insect immunity. Mol Immunol. 106: 69-76. Doi:10.1016/j.molimm.2018.12.021.

[52] Wang XL, Wang KL, He YY, Lu XR, Wen DH, Wu CF, Zhang JH, Zhang R. (2017) The functions of serpin-3, a negative-regulator involved in prophenoloxidase activation and antimicrobial peptides expression of Chinese oak silkworm, Antheraea pernyi. Dev Com Immunol. 69: 1-11. Doi:10.1016/j.dci. 2016.11.022

[53] Wang Y, Jiang HB, (2017) Prophenoloxidase activation and antimicrobial peptide expression induced by the recombinant microbe binding protein of Manduca sexta. Insect Biochem Mol Biol. 83: 35-43. Doi:10.1016/j.ibmb.2016.10.006.

[54] Whitten, M.M.A., Coates, C.J. (2017) Re-evaluation of insect melanogenesis research: views from the dark side. Pigment cell Melano Res. 30: 386-401. Doi: 10. 1111 /pcmr.12590.

[55] Wu K, Yang B, Huang W, Leonard D, Song HS, Ling EJ. (2016) Gut immunity in Lepidop- teran insects. Dev Com Immunol, 64: 65-74, Doi: 10.1016/j.dci.2016. 02.010

[56] Wu P, Han S, Chen T, Qin G, Li L, Guo X. (2013) Involvement of microRNAs in infection of silkworm with Bombyx mori cytoplasmic polyhedrosis virus (BmCPV). PLoS ONE, 8(7): e68209. Doi:10.1371/journal.pone.0068209.

[57] Wu, K., Zhang, J., Zhang, Q., Zhu, S., Shao, Q., Clark, K.D., Liu, Y., Ling, E., (2015) Plant phenolics are detoxified by prophenoloxidase in the insect gut. Sci Rep. 5:16823. Doi: $10.1038 /$ srep 16823. 
[58] Zhang SD, Shen ZJ, Li Z, Wu FM, Zhang BY, Liu YJ, Zhang QW, Liu XX. ( 2015) Identification of a thioredoxin peroxidase gene involved in resistance to nucleopolyhedrovirus infection in Helicoverpa armigera with RNA interference. J Insect Physiol. 82: 17-27. Doi:10.1016/j.jinsphys.2015.07.017.

[59] Zhan XM, Liu HJ, Miao YG, Liu WP. (2006) A comparative study of rac- and Smetolachlor on some activities and metabolism of silkworm, Bombyx mori L. Pesti Biochem Physiol. 85: 133-138.Doi:10.1016/j.pestbp.2005.12.003.

[60] Zhang XL, Guo R, Kumar D, Ma HY, Liu JB, Hu XL, Cao GL, Xue RY, Gong CL. (2015) Identification, gene expression and immune function of the novel Bm-STAT gene in virus-infected Bombyx mori. Gene, 577(1): 82-88. Doi:10.1016/j.gene.2015. 11.027.

[61] Zhao GD, Huang MX, Zhang YL, Wang XC, Du J, Li B, Chen YH, Xu YY, Shen WD, Wei ZG. (2014 ) Expression analysis and RNA interference of BmCarE-10 gene from Bombyx mori. Mol Biol Rep. 41:1607-1616. Doi: 10.1007/s11033-013-3007-3.

[62] Zhao P, Wang GH, Dong ZM, Duan J, Xu PZ, Cheng TC, Xiang ZH, Xia QY, (2010) Genome-wide identification and expression analysis of serine proteases and homologs in the silkworm Bombyx mori. BMC Gen. 11:405. Doi:10.1186/1471- 2164-11-405.

[63] Zhou XS, Chen C, Li TH, Tang JJ, Zhu BJ, Wei GQ, Qian C, Liu CL, Wang L. (2019) A QM protein from Bombyx mori negatively regulates prophenoloxidase activation and melanization by interacting with Jun protein. Insect Mol Biol. 28:1-13. Doi: 10.1111/imb.12573.

[64] Zou FM, Lee KS, Kim BY, Kim HJ, Gui ZZ, Zhang GZ, Guo XJ, Jin BR. (2015) Differential and spatial regulation of the prophenoloxidase (proPO) and proPO-activating enzyme in cuticular melanization and innate immunity in Bombyx mori pupae. J AsiaPacific Entomol. 18: 757-764. Doi:10.1016/j.aspen.2015.09.007. 